\title{
Age and interhemispheric transfer time: a failure to replicate
}

\author{
Elie Ratinckx, Marc Brysbaert*, Géry d'Ydewalle \\ University of Leuven, Leuven, Belgium
}

Accepted 26 November 1996

\begin{abstract}
In a recent study with the Poffenberger paradigm, Brizzolara et al. reported longer estimates of interhemispheric transfer time (IHTT) for children aged 7 years than for adults. They interpreted this finding as evidence for incomplete functional maturity of the corpus callosum in young children. The present study was we were unable to replicate the age effect reported by Brizzolara et al. A closer look at the original study revealed that only 80 observations per child had been collected, which makes it probable that the larger IHTTs in 7-year-olds were caused by stimulus-response compatibility rather than by the lower efficiency of the corpus callosum during childhood years. (C) 1997 Elsevier Science B.V.
\end{abstract}

Keywords: Interhemispheric transfer time; Corpus callosum; Stimulus-response compatibility

\section{Introduction}

It is well documented that the corpus callosum undergoes a long growth process in overall size from birth throughout childhood. There is a rapid growth until the age of 3 years, and a much slower increase afterwards that continues until adolescence [14,21]. The growth is virtually entirely due to a myelination process. At birth, the corpus callosum appears unmyelinated, while in the human adult approximately $40 \%$ of the axons are myelinated [2]. Myelination is nearly complete around age 10 [22].

It has been hypothesized that the unmyelinated corpus callosum puts a newborn baby in a sort of 'physiological disconnection syndrome'. For instance, De Schonen and Bry [12] presented infants of 12-26 weeks with a visual category discrimination learning task

\footnotetext{
* Corresponding author. Laboratory of Experimental Psychology, University of Leuven, B-3000 Leuven, Belgium. Fax: + 3216326099 ; e-mail: Marc.Brysbaert@psy.kuleuven.ac.be
}

(normal versus scrambled faces) in the right or left visual field. Once the infants had reached the learning criterion, they were tested for transfer to the untrained visual field in order to establish whether visual experience could be communicated to the opposite hemisphere. Transfer of learning was found to occur in infants aged 19-26 weeks but not in younger ones.

Brizzolara and colleagues [7] recently extended this work on the development of callosal transmission by looking at the performance of primary school children on the Poffenberger task [19]. In this task, subjects are asked to make unimanual responses to light flashes presented left or right of the fixation location. The usual finding is that responses with the hand contralateral to the stimulated visual hemifield (i.e., crossed responses) are slower than responses with the hand on the same side of the stimulated visual field (uncrossed responses). The difference between crossed and uncrossed responses (CUD) is taken as a measure of interhemispheric transfer time (IHTT). For normal adults, its value varies between 1 and $10 \mathrm{~ms}$, and has an average of $3.8 \mathrm{~ms}$ (for reviews, see $[3,6,17]$ ). The CUD is considerably larger for subjects with section of the 
corpus callosum $(M=54.8 \mathrm{~ms})$ and for subjects with agenesis of the corpus callosum $(M=17.7 \mathrm{~ms})[11,17]$.

In agreement with the idea of reduced interhemispheric transfer in children, Brizzolara et al. [7] reported an age-related decrease of IHTT from age 7 till age 9. The CUD was $21.5 \mathrm{~ms}$ for 7-year olds, whereas normal, 'adult-like' CUDs of 5.8 and $6.6 \mathrm{~ms}$ were obtained for 9- and 11-year olds respectively.

The present study was intended to further investigate the properties of the 'physiological disconnection syndrome' in young children by looking at the effects of stimulus intensity. Acallosals and split-brain patients not only show prolonged IHTTs in the Poffenberger paradigm; their CUD is also a function of the brightness of the stimulus presented in the left and the right visual field. They have a larger CUD for faint light flashes than for bright light flashes [11]. This property is not shared by normal individuals whose CUD remains stable over a wide range of intensities [9,11]. We hypothesized that if the $21 \mathrm{~ms}$ CUD of 7-year old children has the same origin as the $18 \mathrm{~ms}$ CUD of acallosals, then it should similarly be affected by the brightness of the light flash. This was tested by comparing the CUDs of 7- and 11-year old children on three different stimulus intensities.

\section{Method}

\subsection{Subjects}

Sixty children took part in the experiment. They were pupils of a middle-class school in the centre of Antwerp. All subjects were boys. Handedness was assessed with a translation of the Oldfield [18] questionnaire. All but five children were right-handed. The subjects consisted of two age groups: a 7-year old group (mean age $=6.6$ years, range $=6.1-7.9)$ and an 11-year old group (mean age $=11.3$ years, range $=11.1-13$ ) None of the children had neurological, emotional or learning problems, nor at the time of testing nor in their previous history. All children were unaware of the purpose of the experiment and had normal or corrected-to-normal vision.

\subsection{Procedure}

The stimuli were generated with an IBM XT microcomputer. The computer made use of a Hercules monochrome graphics card that had a resolution of $720 \times 348$ pixels, and was connected to a black-andwhite LASER monitor which subtended an area of $255 \times 194 \mathrm{~mm}$. Measurement of the luminance of the screen with a luminance meter (Minolta NT-1) revealed that the right side of the screen was slightly brighter than the left side. This was true for all CRT screens we tested and probably reflects a characteristic of the phosphor beam. Since the difference in brightness could have an effect on the reaction times, the screen was rotated 180 degrees for half of the subjects (betweensubjects condition). (It should be noted that turning a screen upside down makes that a stimulus which was initially programmed to appear right of the fixation location, actually appears in the LVF of the subject, and vice versa. This feature was overlooked in the first analysis we reported on the experiment [20], 50 that we erroneously took a good counterbalancing for an unreliable CUD. This explains the divergence between the present results and those mentioned in [20].) The luminance of the screen was adjusted to $36 \mathrm{~cd} / \mathrm{m}^{2}$. Responses were measured with an external response button connected to the parallel port of the microcomputer. Stimulus and response timing happened to the nearest ms using software routines published by Brysbaert and colleagues [5,8,10].

Stimuli were light flashes presented $75 \mathrm{~mm}$ left or right of a central fixation point that consisted of two short vertical lines of three pixels separated by a gap of 9 pixels. Subjects were instructed to fixate the gap. The children were sitting on a distance of about $60 \mathrm{~cm}$ from the screen (there were no head restraints). At a distance of $57 \mathrm{~cm}$, stimulus magnitude of $10 \mathrm{~mm}$ corresponds with a visual angle of $1^{\circ}$. Stimulus intensity was manipulated by varying the number of pixels of the light flash. The faintest stimulus consisted of $2 \times 2$ pixels, followed respectively by light flashes of $4 \times 4$ and $8 \times 8$ pixels. The experiment was carried out in a quiet room of the school in almost full darkness: only a small light source was put underneath the table on which the computer was placed. This was done to avoid that the children would feel too uncomfortable with an unfamiliar experimenter in the same room.

A trial started by a foreperiod of $1000 \mathrm{~ms}$, followed by an auditory warning signal. After a second random foreperiod of 400,450,500,550, or $600 \mathrm{~ms}$, the light flash was presented for $60 \mathrm{~ms}$ randomly left or right of the fixation point. The children were instructed to fixate the central mark and to press the response button as fast as possible upon seeing the light flash. The responst device was placed on the midline, and the children had to react unimanually with their index finger. The hand with which the subject reacted was counterbalanced in an ABBA form; the hand with which the subjects started was also counterbalanced. Reaction times on stimulus trials shorter than $100 \mathrm{~ms}$ or longer than 1000 $\mathrm{ms}$ were rejected and repeated at a random place later in the series. The fixation mark remained visible throughout the whole experiment.

Each child went through a training block of 20 trials and four experimental blocks of 60 trials each. This resulted in a total of 20 observations per child on each of the $2 \times 3 \times 2(\mathrm{VHF} \times$ intensity $\times$ hand $)=12$ within- 
subjects conditions. At the end of an experimental block, the subject received information about his mean response latency, his standard deviation and the number of reaction times shorter than $100 \mathrm{~ms}$ or longer than $1000 \mathrm{~ms}$.

\section{Results}

Mean RTs (in milliseconds) of all hand 'stimulus intensity' and visual hemifield combinations were entered in the statistical analysis (see Table 1). A $2 \times 2 \times$ $3 \times 2$ analysis of variance with the variables: age (between-subjects), hand, stimulus intensity and visual hemifield (within subjects) returned the following significant effects. There was a main effect of age: 7-year olds were $71 \mathrm{~ms}$ slower than 11-year olds $(332 \mathrm{~ms}$ versus $261 \mathrm{~ms} ; F(1,58)=39.4, P<0.01)$. There was also a significant main effect of stimulus Intensity, because the weakest stimuli took $18 \mathrm{~ms}$ more to react to than the strongest stimuli (RTs of $306 \mathrm{~ms}, 297 \mathrm{~ms}$, and $288 \mathrm{~ms}$ for the three stimulus intensities in ascending order: $F(2,116)=72.2, P<0.01)$. Finally, there was a significant interaction between hand and visual hemifield $(F(1,58)=6.8, P<0.02)$, due to the fact that crossed responses took $3.5 \mathrm{~ms}$ longer than uncrossed responses. As can be seen in Fig. 1, the difference was more pronounced for the left hand than for the right hand. This is in line with the majority of studies on the Poffenberger paradigm [17]. The interaction between hand and visual hemifield was the same for both age groups (three-way interaction, $F<1$; CUD 7 year $=2.8$ $\mathrm{ms}$, CUD 11 year $=4.2 \mathrm{~ms}$ ).

As the larger CUD for 7-year olds was not present, the predicted interaction between stimulus intensity and crossed/uncrossed responses (see Section 1) could not be verified. There was a tendency towards smaller CUDs for bright stimuli, but this was not reliable $(F(2,116)=2.0, P>0.10)$.

Table 1

Reaction times as a function of age, visual hemifield, stimulus intensity, and responding hand

\begin{tabular}{|c|c|c|c|c|c|c|}
\hline \multirow[t]{3}{*}{ Hand } & \multicolumn{6}{|c|}{ Stimulus intensity } \\
\hline & \multicolumn{3}{|l|}{ LVF } & \multicolumn{3}{|l|}{ RVF } \\
\hline & 1 & 2 & 3 & 1 & 2 & 3 \\
\hline \multicolumn{7}{|c|}{ 7-year olds } \\
\hline Left & 335.9 & 324.2 & 319.6 & 341.2 & 337.3 & 321.0 \\
\hline Right & 348.7 & 327.8 & 327.0 & 342.0 & 341.0 & 323.6 \\
\hline CUD & 12.8 & 3.6 & 7.4 & -0.8 & -2.7 & -2.6 \\
\hline \multicolumn{7}{|c|}{ 11-year olds } \\
\hline Left & 263.7 & 259.2 & 252.8 & 277.2 & 265.4 & 251.7 \\
\hline Right & 270.4 & 262.2 & 253.4 & 267.1 & 256.3 & 255.9 \\
\hline CUD & 6.7 & 3.0 & 0.6 & 10.1 & 9.1 & -4.2 \\
\hline
\end{tabular}

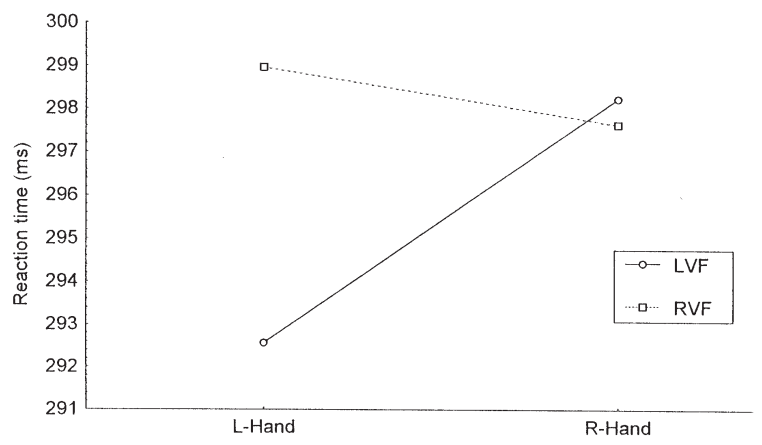

Fig. 1. Interaction between hand and visual hemifield.

\section{Discussion}

In a recent study, Brizzolara et al. [7] reported evidence for a higher IHTT-estimate in 7-year old children than in 11-year olds and adults. They attributed this increase to incomplete maturity of the corpus callosum at the age of 7 years. In the present experiment we investigated whether the higher IHTT-measure for young children also implied that their IHTT estimate is a function of stimulus intensity, as previously demonstrated in split-brain patients and acallosals [11,17], and predicted by the variable-criterion model of Brysbaert [9].

Much to our surprise, however, the finding of Brizzolara et al. could not be replicated in the present study. There was no significant difference in CUD between 7and 11-year old children, and both values were similar to those reported for adults $[3,6,17]$. The following post-hoc explanation may illustrate why this was the case.

When designing a Poffenberger experiment, researchers must be very careful to ensure that the CUDs they obtain reflect interhemispheric transfer time and are not due to the well-known spatial stimulus-response compatibility effect $[13,16]$. For a whole variety of tasks it is easier to respond with the right hand to stimuli presented in the right hemispace and with the left hand to stimuli presented in the left hemispace. This is due to attentional factors as can be concluded from the finding that the effect reverses when the subjects cross their hands across the midline. The stimulus-response compatibility effect has been suggested several times as an explanation of the CUD in the Poffenberger paradigm before, but is usually refuted by making reference to a series of carefully controlled studies by Berlucchi and colleagues $[1,4]$ who found the same CUDs in conditions with crossed and uncrossed hands. What is often overlooked, however, is that the findings of Berlucchi et al. were based on a total of more than 1000 trials per subject and with adults. Brysbaert [9] presented evidence that when the number of trials is smaller and the subjects less experienced, stimulus-response compatibility is likely to confound the CUD estimates. 
In their study, Brizzolara et al. only had 80 observations per child and they did not make reference to any practice trials. So, it is not unlikely that stimulus-response compatibility affected the CUD estimates, and more so for the young children than for the older ones (cf. the estimates of the older children were also in the high range). This would explain why we could not replicate the finding in our study which had a total of 240 observations per subject and a separate training block of 20 trials at the beginning of the experiment.

In conclusion, on the basis of our own data and the re-analysis of the Brizzolara et al. study, we do not believe that children of age seven behave as acallosals in the Poffenberger task. Rather, we think that these children are more susceptible to attentional factors. If they are given enough practice, their IHTT is of the same magnitude as that of adults, i.e. around $3 \mathrm{~ms}$. On the other hand, we also want to stress that we do not consider this finding as hard evidence against the idea that the corpus callosum is not fully developed at the age of seven. As we have argued before [9], the Poffenberger task measures reactions to a very particular event, namely the onset of unstructured light flashes. This processing is likely to be controlled by the magnocellular pathway and not by the parvocellular pathway that underlies object recognition, e.g. [15]. Finding similar IHTTs for young children and adults in the Poffenberger task, then, only points to the fact that these particular connections are equally effective in both groups.

\section{Acknowledgements}

The authors wish to thank the School Director, the teachers and the children of the elementary school of the Sint-Jan Berchmans college in Antwerp.

\section{References}

[1] Anzola, G.P. Bertolini, G. Buchtel, H.A. and Rizzolatti, G., Spatial compatibility and anatomical factors in simple and choice reaction time, Neuropsychologia, 15 (1977) 295-302.

[2] Barkovich, A.J. Lyon, G. and Evrard, P., Formation, maturation, and disorders of white matter, Am. J. Neuroradiol., 13 (1992) 447-461.

[3] Bashore, T.R., Vocal and manual reaction time estimates of interhemispheric transmission time, Psychol. Bull., 89 (1981) $352-368$.
[4] Berlucchi, G. Crea, F. Di Stefano, M and Tassinari, G., Influence of spatial stimulus-response compatibility on reaction time of ipsilateral and contralateral hand to lateralized light stimuli, J. Exp. Psychol. HPP, 3 (1977) 505-517.

[5] Bovens, N. and Brysbaert, M., IBM PC/XT/AT and PS/2 Turbo Pascal timing with extended resolution, Behav. Res. Methods Instr. Comp., 22 (1990) 332-334.

[6] Braun, C.M.J., Estimation of interhemispheric dynamics from simple unimanual reaction time to extrafoveal stimuli, Neuropsychol. Rev., 3 (1992) 321-364.

[7] Brizzolara, D. Ferretti, G. Brovedani, P. Casalini, C. and Sbrana, B., Is interhemispheric transfer time related to age? A developmental study, Behav. Brain Res., 64 (1994) 179-184.

[8] Brysbaert, M., A warning about millisecond timing in Turbo Pascal, Behav. Res. Methods Instr. Comp., 22 (1990) 344-345.

[9] Brysbaert, M., Behavioral estimates of interhemispheric transmission time and the signal detection method: a reappraisal, Percept. Psychophys., 56 (1994) 479-490.

[10] Brysbaert, M. Bovens, N. d'Ydewalle, G. and Van Calster, J., Turbo Pascal timing routines for the IBM microcomputer family, Behav. Res. Methods Instr. Comp., 21 (1989) 73-83.

[11] Clarke, J.M. and Zaidel, E., Simple reaction times to lateralized light flashes: Varieties of interhemispheric communication routes, Brain, 112 (1989) 849-870.

[12] de Schonen, S. and Bry, I., Interhemispheric communication of visual learning: A developmental study in 3-6-month old infants, Neuropsychologia, 25 (1987) 601-612.

[13] Fitts, P.M., Engineering psychology and equipment design. In S. Stevens (Ed.), Handbook of Experimental Psychology, 1951, Wiley, New York, pp. 1287-1340.

[14] Innocenti, G.M., General organisation of callosal connections in the cerebral cortex. In E.G. Jones and A.A. Peters (Eds.), Cerebral Cortex, Vol. 5, 1986, Plenum, New York, pp. 291-353.

[15] Kaas, J.H., Changing concepts of visual cortex organization in primates. In J.W. Brown (Ed.), Neuropsychology of Visual Perception, 1989, Erlbaum, Hillsdale, NJ.

[16] Lamberts, K. Tavernier, G. and d'Ydewalle, G., Simultaneous effects of multiple reference points in spatial stimulus-response compatibility, Acta Psychol., 79 (1992) 115-130.

[17] Marzi, C.A. Bisiacchi, P. and Nicoletti, R., Is interhemispheric transfer of visuomotor information asymmetric? Evidence from a meta-analysis, Neuropsychologia, 29 (1991) 1163-1177.

[18] Oldfield, R.C., The assessment and analysis of handedness: The Edinburgh inventory, Neuropsychologia, 9 (1971) 97-113.

[19] Poffenberger, A.T., Reaction time to retinal stimulation, with special reference to the time lost through nerve centers, Arch. Psychol., 23 (1912) 1-73.

[20] Ratinckx, E. Brysbaert, M. and d'Ydewalle G., Age and interhemispheric transmission time: A failure to replicate. Psychological Reports of the Laboratory of Experimental Psychology 177, 1995, University of Leuven, Leuven, Belgium.

[21] Witelson, S. and Kigar, D.L., Anatomical development of the corpus callosum in humans: A review with reference to sex and cognition. In D.L. Molfese and S.J. Segalowitz (Eds.), Brain Lateralization in Children, 1988, The Guilford Press, New York, pp. 35-57.

[22] Yakovlev, P.1. and Lecours, A., The myelogenetic cycles of regional maturation of the brain. In A. Minkowski (Ed.), Regional Development of the Brain in Early Life, 1967, Blackwell, London, pp. 3-65. 